\title{
Update on Varicose Veins Recurrence and its management
}

\author{
Pittaluga P. ${ }^{1}$, Maeseneer M. ${ }^{1}$, \\ Chastanet S. ${ }^{1}$ \\ ${ }^{1}$ Nice, France. \\ E-mail:paulpittaluga@hotmail.com
}

Pittaluga, P.; Maeseneer, M.; Ấhastanet, S. 2013. Update on Varicose Veins Recurrence and its management, p.Ào. In: Bastos, Francisco Reis. Anais do V Simpósio Internacional de Flebologia [Blucher Medical Proceedings n.1 v.1]. São Paulo: Blucher, 2014

http://dx.doi.org/10.5151/medpro-flebo-SIF_52

\section{Introduction}

Recurrence of varicose veins after treatment has always been a very disappointing phenomenon for patients and surgeons a like. Up to the beginning of the $21^{\text {st }}$ century, treatment of recurrent varicose veins varied between a minimally invasive treatment (mainly liquid sclerotherapy) and a maximally invasive re-intervention, including re-exploration of the groin or popliteal fossa and removal of all recurrent varicose veins. Although the majority of these patients belong to the C2-C3 clinical class of the CEAP classification, more advanced stages of chronic venous insufficiency (C4-C6) have been registered in $29 \%$ of patients presenting with recurrent varicose veins after surgery. ${ }^{1}$ After 5 years clinical recurrence was seen at thigh level in $20 \%$ of 50 limbs after primary versus $42 \%$ of 50 limbs after redo surgery. ${ }^{5}$

\section{Towards less invasive techniques}

Treatment strategy should therefore become far less invasive. Reopening the groin (or popliteal fossa) should be absolutely avoided. Moreover treatment can easily be performed step-by-step instead of all at once, if appropriate. This means treatment can be easily performed in an ambulatory setting under local (tumescent) anesthesia. Several less invasive alternative methods have been proposed during the last years: Phlebectomies without re-opening the groin. In a recently published retrospective study, Pittaluga et al. ${ }^{6}$ compared classic redo surgery including re-exploration of the groin (137 patients) with a minimally invasive procedure under tumescent local anesthesia focusing on the 'varicose reservoir' without re-opening the groin (151 patients). In the latter cases, surgery was limited to multiple phlebectomies, trying to remove all varicose veins from the subcutis. This technique is based on the 'ascending' pathophysiologic theory, which states that varicose disease (and hence reflux) in most cases starts at the level of superficial tributaries rather than at the level of the SFJ, saphenopopliteal junction (SPJ) or junctions of perforating veins with the deep venous system, which are considered as the classic 'sources' of reflux in the 'descending' theory. The authors reported good mid-term results after 3 years, less complications and a much lower cost for this minimally invasive procedure. It can be concluded that this strategy is certainly applicable in many patients with recurrent varicose veins.

Limited redo surgery with peroperative foam sclerotherapy and endo- 
venous thermal ablation (EVTA) of residual GSV/AASV/SSV trunk with laser or radiofrequency energy.

In conclusion, endovenous ablation of a residual refluxing GSV, AASV or SSV trunk should be considered a valuable and safe alternative for redo surgery at the SFJ or SPJ combined with stripping of the residual trunk.

\section{Ultrasound guided Foam sclerotherapy (UGFS)}

Probably UGFS is the technique most widely used nowadays to treat recurrent varicose veins. In a survey in the UK, $71 \%$ of 281 vascular surgeons answered they would use foam sclerotherapy in case of recurrent varicose veins. Kakkos et al. reported excellent immediate results after treating 45 legs with recurrent varicose veins using $3 \%$ sodium tetradecyl sulphate foam. Only in $58 \%$ of cases a single injection session was sufficient whereas the others needed at least 2 treatment sessions. Reviewing the problem of recurrent varices at the popliteal fossa Perrin et al. ${ }^{16}$ recommended UGFS as the treatment of choice unless duplex ultrasound showed an incompetent stump at the SPJ with a gross reflux filling the recurrent venous network. In very exceptional cases redo surgery in experienced hands might still be an option, although extensive phlebectomies alone should always be considered even in these extensive cases (see above). In the vast majority of cases however UGFS is a very valuable option.

\section{Treatment of pelvic vein incompetence}

Phlebography demonstrated extension of reflux into varicose veins of the groin or limb in 44 patients (62\%). If these findings can be confirmed this might become a simple tool to identify patients with recurrent varicose veins who could potentially benefit from treatment of their pelvic vein incompetence.

\section{Conclusion}

Classic redo surgery to treat varicose vein recurrence is no longer an option in the vast majority of patients, considering all the minimally invasive methods that have been developed in recent years and are easily available today. In order to decide which technique will be appropriate for an individual patient, a well performed duplex ultrasound scan is mandatory and will be the GPS for an adequate treatment. More well designed prospective studies are certainly needed to validate the above cited alternative methods. However, setting up a RCT to compare one of these new techniques with classic redo surgery does not seem to be justified anymore.

Key-words: UGVS, treatment of varicose veins, phlebectomy, foam sclerotherapy, recurrence. 